\title{
COMMISSIONING OF AN APPLE-II UNDULATOR AT DARESBURY LABORATORY FOR THE SRS
}

\author{
B.J.A. Shepherd", D.J. Scott, F.E. Hannon, N.G. Wyles, J.A. Clarke, ASTeC, CCLRC Daresbury \\ Laboratory, UK
}

\section{Abstract}

A new variable polarisation undulator of the APPLE-II type has been designed and constructed at Daresbury Laboratory. Initial magnet testing of the $56 \mathrm{~mm}$ period device was followed by an intensive period of shimming to improve the field quality. After this was successfully completed the undulator was installed into the SRS and tests made of the effect of the device upon the electron beam. This beam commissioning was completed in a very short space of time, and the beamline group were given full control of the gap and phase of the magnet within a few weeks of installation. This paper summarises the measurement of the magnet and the shimming techniques employed to improve the field quality. It also describes the effect of the device upon the stored $2 \mathrm{GeV}$ electron beam and the measures taken to minimise these effects during user operations.

\section{INTRODUCTION}

HU56 is a new variable-polarisation undulator for the SRS [1]. The device has 17 periods of 56mm each, and has four arrays in the APPLE-II configuration (Figure 1). Two diagonally opposing arrays can be moved in the longitudinal direction with respect to the other arrays. The arrays can be moved in either mutual mode (both in the same direction) or opposing mode (in opposite directions). The undulator phase refers to the amount that the arrays can be moved; this is variable between $\pm 28 \mathrm{~mm}$ (i.e. half a period). The polarisation of the light can thus be varied from pure horizontal or vertical, linear, elliptical or circular.

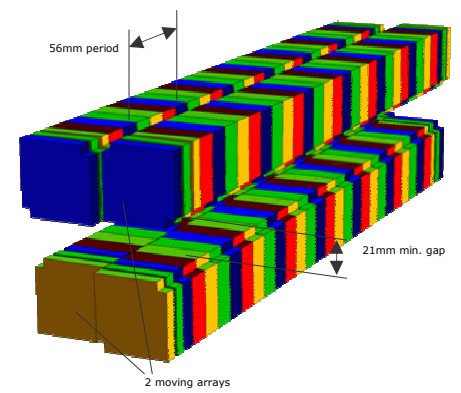

Figure 1: HU56 schematic.

\section{FIELD MAPPING}

A simulated annealing algorithm was used to determine the best sort order for the blocks, given the magnetisation strength and direction of each one [2]. The undulator was built at Daresbury Laboratory's dedicated magnet assembly facility.

"\#.shepherd@dl.ac.uk
A moving Hall probe was used to generate a field map of the undulator, and from this field map a plot of the trajectory of the electron beam through the device could be generated.

Figure 2 shows the first results from the Hall probe scans. This scan was performed on axis, at minimum gap and zero phase, so that the field is purely vertical with a peak of about $0.6 \mathrm{~T}$.

The data was integrated to show the shape of the trajectory through the device. The predicted trajectory was not ideal, so some modification of the device was required to improve this.

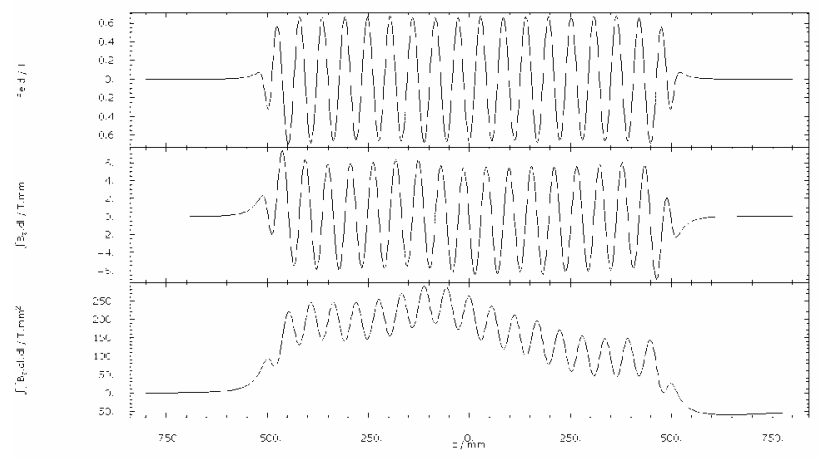

Figure 2: Initial Hall probe scan, integrated to show the trajectory of an electron.

\section{SHIMMING}

Several different methods of correcting the field errors were employed. These included:

- rotating the horizontal blocks by $180^{\circ}$ about the undulator axis

- swapping adjacent vertical blocks and rotating by $180^{\circ}$, and

- swapping both horizontal and vertical blocks out for spare blocks.

The effect of these changes was modelled in Radia [3] in order to assess whether a particular modification would have a beneficial effect or not. Each change was usually carried out on blocks in all four arrays at the same time.

We also considered moving blocks within the arrays, by up to $\pm 200 \mu \mathrm{m}$ at a time in the transverse plane. However, the effect of this 'virtual shimming' was so small that many tens of movements would have been required to effectively improve the trajectory. This amount of correction was not feasible within the available time.

The process of swapping blocks and retesting the undulator was iterated several times. The before-and-after results can be seen in Figure 3. 


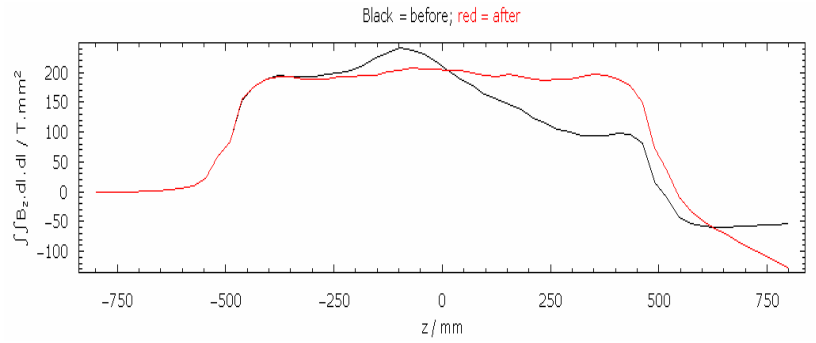

Figure 3: Electron trajectory through the undulator, before (black) and after (red) shimming. Note that the wiggle motion is smoothed out in this plot for clarity.

The trajectory in vertical field mode (phase $=0$ ) shows a big improvement from the original measurements. The exit angle (overall field integral) is quite large, but this was more accurately measured using the flipping coil bench. The on-axis phase error was reduced from an initial value of $9.0^{\circ}$ down to $4.4^{\circ}$.

A 3-axis Hall probe was used to measure the vertical and horizontal field at the same time, at minimum gap and all phases in mutual mode. This allows us to find the point at which $\mathrm{H}$ and $\mathrm{V}$ peak fields are equal, yielding circularly polarised light. Figure 4 shows the result.

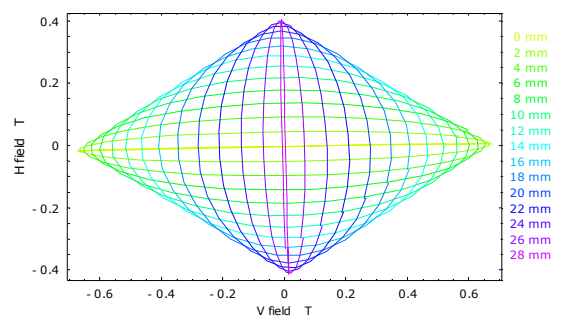

Figure 4: $\mathrm{H}$ and $\mathrm{V}$ fields for different phases, at minimum gap $(21 \mathrm{~mm})$.

The peak $\mathrm{H}$ and $\mathrm{V}$ fields are $0.65 \mathrm{~T}$ and $0.4 \mathrm{~T}$ respectively, and the peak fields are equal $(0.33 \mathrm{~T})$ at phase $=18.1 \mathrm{~mm}$ as expected.

\section{FIELD INTEGRALS}

The field integrals were measured using a flipping coil. The aim here was to measure the integrals as a function of transverse position, gap and phase. These need to be reduced as far as possible, to minimise the effect on the stored beam. The range of the trim coils was tested first. It was found that at minimum gap, the trim coils could correct field integrals of up to $\pm 600 \mathrm{~T} . \mu \mathrm{m}$ in both horizontal and vertical directions, by powering them at the maximum current of $\pm 7 \mathrm{~A}$. This gives us a minimum requirement for reducing the field integrals by moving blocks.

The 'virtual shimming' technique (moving blocks in the transverse plane) was used again, this time moving blocks by up to $0.5 \mathrm{~mm}$. The gap of the device was reduced by $1 \mathrm{~mm}$ but this was not a problem since the magnet blocks were stronger than expected. Blocks at the exit of the device were moved to avoid any detrimental effects on the overall trajectory.
A Radia model was used to determine the optimum type and number of moves to make - a database of shimming 'profiles' was built up, enabling moves to be combined to find the best ones to improve both the horizontal and vertical integrals. This is not an intuitive problem as one single change tends to affect horizontal and vertical fields in different ways. However, the changes predicted by the model matched the observed data very well.

Figure 5 shows a summary of the overall changes to the integrals.
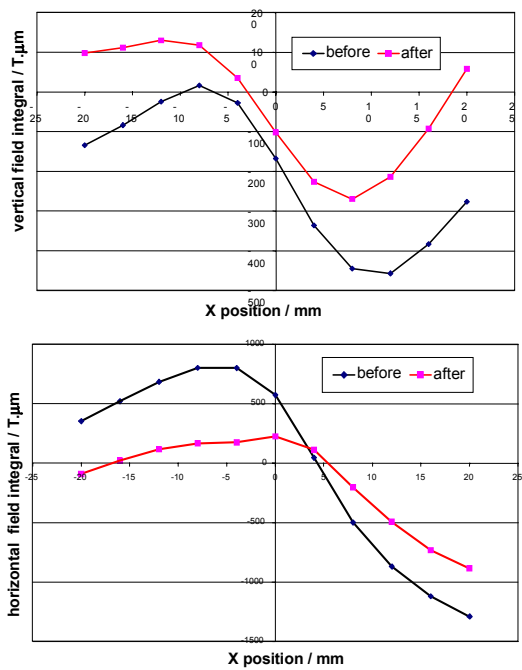

Figure 5: Improvements to the field integrals as measured by the flipping coil (black = before; pink $=$ after).

The integrals were reduced to within the limits defined by the trim coils in most cases. The overall effect on the beam in terms of dipole and sextupole components was also reduced. Given more time a better result could have been obtained. The results are similar to those obtained elsewhere $[5,6]$.

\section{COMMISSIONING}

Undulator commissioning with beam was carried out during dedicated beam physics periods, with a $2 \mathrm{GeV}$ multibunch beam and all other insertion devices wound in or powered up [6]. Commissioning of the device took around 6 machine physics days altogether.

One major task was to set up the lookup tables for the trim coils. This is necessary since the beamline users needed full control of the undulator gap and phase in order to control the polarisation of the output light. The trim coils are automatically adjusted whenever the gap and phase are changed to compensate for field integrals and ensure the beam is not disturbed for other users. For each gap/phase point, the optimum trim coil currents must be found to minimise the RMS beam position in the machine, as measured by the photon beam tungsten vane monitors (TVMs).

Figure 6 illustrates the effect of the undulator on the photon beam position without the use of the trim coils. There are several large regions where the RMS beam position goes up to a few hundred $\mu \mathrm{m}$. 


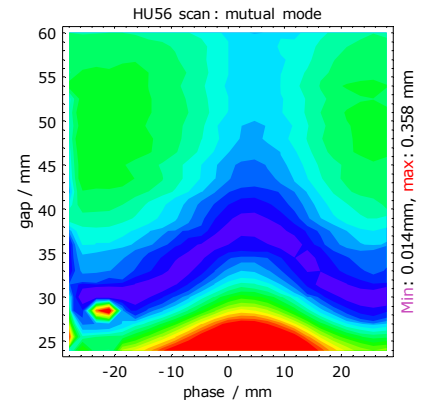

Figure 6: RMS beam position without using trim coils, as a function of HU56 gap (vertical axis) and phase (horizontal axis).

This scan gives us a general idea of how much correction is required at each gap/phase point. The track map table was then filled in by hand, using a spreadsheet to translate a movement of the beam to trim current values. The aim was to keep the RMS as low as possible, generally under $10 \mu \mathrm{m}$. The results of this are shown in Figure 7.
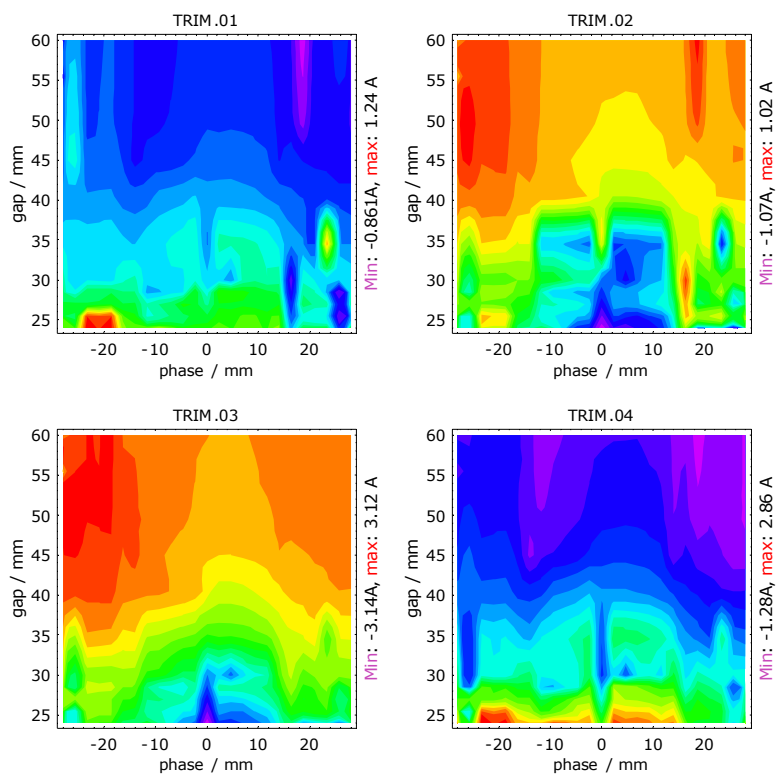

Figure 7: Trim coil currents required to keep the beam steady for each gap/phase setting of HU56.

The current demand for each coil generally stays fairly low, and is between $\pm 3 \mathrm{~A}$ in all cases. This is well within the coil capacity of $\pm 7 \mathrm{~A}$.

The gap/phase scan was then repeated, this time with the trim coils powered and following the track map. The new RMS beam positions are shown in Figure 8.

The beam is shown to be much more stable using the trim coils. There is a slight drift with gap; this is simply due to the slow drift of the beam due to thermal effects (automatic correction was switched off for the duration of the scan). Beam movement with undulator phase is reduced to a few $\mu \mathrm{m}$.

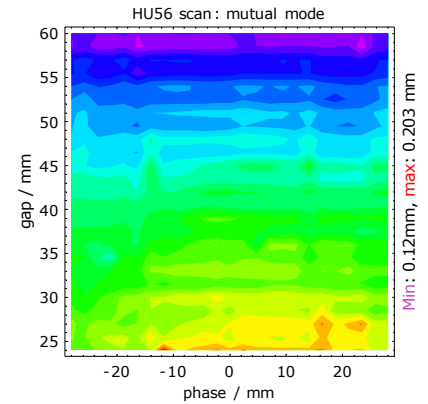

Figure 8: RMS beam position using trim coils, as a function of gap and phase.

A shunt quadrupole is available in the undulator straight to correct for any tune shifts. However, the tune shifts due to the undulator were measured and found to be negligible, so the shunt will not be used.

Currently a track map only exists for mutual mode. The task will be repeated for opposing mode.

In January 2005, an experiment was run on the beamline with the undulator set to produce circularly polarised light at a photon energy of $274 \mathrm{eV}$ (first harmonic). The amount of polarisation was measured as -0.935 , in line with expectations [7].

\section{CONCLUSIONS}

A new insertion device has been installed and commissioned in the SRS. Testing and shimming of the device, though tightly constrained by time, was completed successfully.

Commissioning with beam has also taken place successfully. In mutual mode, full control of the gap and phase has already been handed over to the users, and experiments have been carried out which demonstrate the circularly polarised light output. The undulator has no effect on the beam lifetime or coupling of the horizontal and vertical emittances.

Opposing mode commissioning is planned for the next beam physics periods during May and June 2005.

\section{REFERENCES}

[1] F.E. Hannon et al, 'Construction of an APPLE-II Type Undulator at Daresbury Laboratory for the SRS', EPAC 2004, p. 440.

[2] D.J. Scott, 'Magnet Block Sorting For Variably Polarising Undulators', EPAC 2004, p. 461.

[3] Radia (magnet modelling code), http://www.esrf.fr/machine/groups/insertion_devices/ Codes/Radia/Radia.html.

[4] B. Diviacco et al, 'Development Of Elliptical Undulators For ELETTRA', EPAC 2000, p. 2322.

[5] S. Marks et al, 'Magnetic Performance Of The Advanced Light Source EPU5.0 Elliptically Polarizing Undulator', PAC 1999, p. 162.

[6] D.J. Scott, 'Initial Commissioning Results for HU56', SRS/APES/04/07, 2 December 2004.

[7] M.A. MacDonald, private communication. 